\title{
MiR199a is implicated in embryo implantation by regulating Grb10 in rat
}

\author{
Hong-Fei Xia ${ }^{1,2}$, Jing-Li Cao ${ }^{1,2}$, Xiao-Hua Jin ${ }^{1}$ and Xu Ma ${ }^{1,2}$ \\ ${ }^{1}$ Reproductive and Genetic Center of National Research Institute for Family Planning, Beijing 100081, China and \\ ${ }^{2}$ Graduate School, Peking Union Medical College, Beijing 100730, China
}

Correspondence should be addressed to X Ma; Email: genetic@263.net.cn or to H-F Xia; Email: hongfeixia@126.com

\begin{abstract}
MiR199a was found to be differentially expressed in rat uteri between the prereceptive and receptive phase via microRNA (miRNA) microarray analysis in our previous study. However, the role of miR199a in rat embryo implantation remained unknown. In the study, northern blot results showed that the expression levels of miR199a were higher on gestation days 5 and 6 (g.d.5-6) in rat uteri than on g.d.3-4 and g.d.7-8. In situ localization of miR199a in rat uteri showed that miR199a was mainly localized in the stroma or decidua. The expression of miR199a was not significantly different in the uteri of pseudopregnant rats and evidently increased in the uteri of rats subjected to activation of delayed implantation and experimentally induced decidualization. Treatment with $17 \beta$-estradiol or both $17 \beta$-estradiol and progesterone significantly diminished miR199a levels. Gain of function of miR199a in endometrial stromal cells isolated from rat uteri inhibited cell proliferation and promoted cell apoptosis. Loss of function of miR199a displayed opposite roles on cell proliferation and apoptosis. Further investigation uncovered a significant inverse association between the expression of miR199a and growth factor receptor-bound protein 10 (Grb10), an imprinted gene, and miR199a could bind to the $3^{\prime}$ UTR of Grb10 to inhibit Grb10 translation. In addition, in vivo analysis found that the immunostaining of GRB10 was attenuated in the stroma or decidua from g.d.4 to 6, contrary to the enhancement of miR199a. Collectively, upregulation of miR199a in rat uterus during the receptive phase is regulated by blastocyst activation and uterine decidualization. Enforced miR199a expression suppresses cell proliferation partially through targeting Grb10.
\end{abstract}

Reproduction (2014) 147 91-99

\section{Introduction}

Pregnancy begins with the fertilization of the ovum, followed by implantation of the blastocyst into the maternal uterus (Carson et al. 2000, Koot \& Macklon 2013). Implantation is an essential step in the development of a pregnancy (Koot \& Macklon 2013). Blastocysts can implant only if the endometrium has been induced, a transient state of endometrial receptivity for embryo implantation that lasts for $<24 \mathrm{~h}$ (Quinn et al. 2006, Granot et al. 2012). Endometrial receptivity for embryo implantation in rats occurs on day 5 of pregnancy. The time surrounding the window of receptivity is referred to as the peri-implantation period. In response to implanting embryos, the underlying endometrial stromal cells (ESCs) undergo decidualization (Zhang et al. 2012b). Although a large number of studies have been conducted to explore the exact molecular mechanisms of embryo implantation, there are many unanswered questions about the specific mechanisms associated with the onset of uterine receptivity and embryo implantation.

MicroRNAs (miRNAs) are short noncoding RNAs that are involved in several important biological processes through the regulation of gene expression at the posttranscriptional level (Pan et al. 2013, Wang et al. 2013b). The miRNAs elicit their effects by binding to the $3^{\prime}$ UTR of their target mRNAs, leading to the inhibition of translation or the degradation of the mRNA, depending on the degree of complementary base pairing (Guo et al. 2013, Han et al. 2013, Wang et al. 2013a). Our previous study found that miR199a was differentially expressed in rat uteri between the prereceptive and receptive phase by miRNA microarray analysis. MiR199a* was spatiotemporally expressed in mouse uterus during embryo implantation (Chakrabarty et al. 2007). However, the expression and function of miR199a in embryo implantation in rats is still unknown.

In this study, we report the spatiotemporal profiles of miR199a in rat uterus during peri-implantation and the effects of pseudopregnancy, artificial decidualization, activation of delayed implantation, and hormone treatment on miR199a expression. In addition, we also test the effects of miR199a on cell growth and report the identification of growth factor receptor-bound protein $10(\mathrm{Grb10})$ as a regulatory target of miR199a. 


\section{Materials and methods}

\section{Experimental animals and protocols}

Sexually mature, healthy female Sprague-Dawley rats (220-260 g body weight) were purchased from the Laboratory Animal Center of the Academy of Military Medical Sciences (Beijing, China). Rats were housed in a temperature- and humidity-controlled room with a $12 \mathrm{~h}$ light: $12 \mathrm{~h}$ darkness cycle. All animal procedures were approved by the Institutional Animals Care and Use Committee of the National Research Institute for Family Planning. Rats were caged overnight with fertile males of the same strain. The presence of a vaginal plug or sperm was considered as day 1 of gestation (g.d.1). Uteri were excised from g.d.3 to 8 in rat and fixed with $4 \%$ paraformaldehyde (PFA) solution (Sigma-Aldrich) for in situ hybridization or frozen in liquid nitrogen for RNA analysis.

Pseudopregnancy was induced by caging adult females with vasectomized males and mating was confirmed by checking for a vaginal plug (day 1 of pseudopregnancy). The uteri were collected from day 3 to day 7 of pseudopregnancy. On day 5 of pseudopregnancy, when the uteri were optimally sensitized for artificial deciduogenic stimuli, $100 \mu$ olive oil (Sigma-Aldrich) was infused into the lumen of one of the uterine horns to induce artificial decidualization. The contralateral uterine horn, which was not infused with oil, served as a control. At day 7 of pseudopregnancy, the rats were killed and the uterine horns were isolated.

To induce delayed implantation, the pregnant rats on g.d.4 were ovariectomized and injected with progesterone $(5 \mathrm{mg} / \mathrm{rat}$, s.c.; Sigma-Aldrich) intraperitoneally to maintain delayed implantation from g.d.5 to 7 . Then the rats were treated with $17 \beta$-estradiol $(0.5 \mu \mathrm{g} / \mathrm{rat}$; Sigma-Aldrich) to terminate delayed implantation. The rats were killed by stunning and cervical dislocation to collect uteri at $24 \mathrm{~h}$ after estrogen treatment. The implantation sites were also identified by i.v. injection of Chicago blue solution (Sigma-Aldrich). To confirm that the rats receiving progesterone only were in a state of delayed implantation, uterine flushings were collected from g.d.5 to 7 and examined for the presence of hatched blastocysts.

To test the effects of steroid hormones on miR199a expression, treatment with hormones was started 2 weeks after rats had been ovariectomized. The ovariectomized rats were injected with $17 \beta$-estradiol $(1 \mu \mathrm{g} / \mathrm{rat})$ or progesterone $(10 \mathrm{mg} / \mathrm{rat})$ at intervals of $24 \mathrm{~h}$ for 3 days. All steroids were dissolved in olive oil and injected intraperitoneally. The ovariectomized rats were injected with olive oil only $(0.1 \mathrm{ml} / \mathrm{rat})$ as controls.

\section{Northern blot analysis}

Total RNA was isolated from uterus with TRIzol reagent (Invitrogen). Aliquots of $40 \mu \mathrm{g}$ of total RNA per sample were subjected to electrophoresis on a $15 \%$ urea-PAGE gel and transferred to a nylon membrane (Hybond $\mathrm{N}+$; Amersham Pharmacia Biotech). After being u.v. cross-linked and baked at $50{ }^{\circ} \mathrm{C}$ for $30 \mathrm{~min}$, the membrane was prehybridized with hybridization buffer at $42{ }^{\circ} \mathrm{C}$ for $4 \mathrm{~h}$ and then hybridized with ${ }^{32} \mathrm{P}$-labeled miR199a probe (GAACAGGTAGTCTGAACACTGGG) or U6 probe (CGTTCCAATTTTAGTATATGTGCTGCCGAAGCGA) at $40^{\circ} \mathrm{C}$ overnight.
Membranes were washed and exposed to Phosphorlmager screens (GE Healthcare Bio-Sciences Corp., Piscataway, NJ, USA). The bands were analyzed using Quantity One software (Bio-Rad). All experiments were repeated at least three times.

\section{In situ hybridization}

Sections of uterus $(5 \mu \mathrm{m})$ were treated with proteinase $\mathrm{K}$ $(20 \mathrm{~g} / \mathrm{ml})$ for $15 \mathrm{~min}$ and refixed in 4\% PFA for $15 \mathrm{~min}$. After acetylation with $0.25 \%$ acetic anhydride in $0.1 \mathrm{M}$ triethanolamine ( $\mathrm{pH}$ 8.0) for $10 \mathrm{~min}$, sections were prehybridized with hybridization buffer (Roche) at $40{ }^{\circ} \mathrm{C}$ for $2 \mathrm{~h}$ and then hybridized with digoxigenin (DIG)-labeled LNA-miR199a probe (LNA-miR199a sequence: 5'-DIG-gAacagGtAgTctGaAcacTggg- $\left.3^{\prime}\right)$ at $40{ }^{\circ} \mathrm{C}$ overnight. The sections were then incubated in buffer containing anti-DIG-antibody for $2 \mathrm{~h}$ at $37^{\circ} \mathrm{C}$, followed by staining with 5 -bromo-4-chloro-3-indolyl phosphate and $p$-nitroblue tetrazolium chloride (Promega). The sections were hybridized with a DIG-labeled LNA-scrambled probe (LNA-scrambled sequences: 5'-caTtaAtgTcGgaCaaCtCAat- $\left.3^{\prime}\right)$ as a negative control. The sections were observed with an Eclipse 80i microscope (Nikon, Tokyo, Japan).

\section{Cell viability assay}

In vitro cell viability was estimated with an MTS assay. Briefly, ESCs isolated from rat uteri (5000 cells/well) were seeded in 96-well plates (Costar, Corning, Inc., Coring, NY, USA) in DMEM/F12 culture, and allowed to attach overnight. The cells were then transfected with miR199a mimics, premiR control, miR199a inhibitor, and antimiR control respectively. MiR199a mimics are chemically synthesized, double-stranded RNAs that mimic mature endogenous miR199a after transfection into cells. MiR199a inhibitors are chemically synthesized, single-stranded, modified RNAs that specifically inhibit endogenous miR199a function after transfection into cells. Twenty microliters MTT ( $5 \mathrm{mg} / \mathrm{ml}$; Sigma-Aldrich) were added to each well $48 \mathrm{~h}$ after transfection, and incubated for $4 \mathrm{~h}$. Media were then removed and $150 \mu \mathrm{l}$ dimethyl sulfoxide (Sigma-Aldrich) was added in each well. Absorbance was recorded at A570 nm with a 96-well plate reader (Bio-Rad 3550). In order to avoid the error related to the absorbance of the replicated wells, each treatment group was replicated in three wells. In order to decrease the experimental error and calculate the s.D. for each group, the experiment was repeated for three times. In every experiment, the mean absorbance of the replicate wells was calculated and then normalized with the mean absorbance of the control. The results were described as a ratio of A570 $\mathrm{nm}$ with miR199a mimics vs premiR control or miR199a inhibitor vs antimiR control.

\section{Flow cytometry analysis}

Cell apoptosis was performed with flow cytometry analysis by Annexin V-FLUOS staining Kit (Roche), containing $2.5 \mu \mathrm{l}$ annexin V-FITC stock and $5 \mu \mathrm{l} 20 \mu \mathrm{g} / \mathrm{ml}$ phosphatidylinositol $(\mathrm{Pl})$ to determine the phosphatidylserine exposure on the outer plasma membrane. After incubation for $15 \mathrm{~min}$ at room temperature in a light-protected area, the specimens were quantified by flow cytometry (BD Biosciences, San Jose, 
CA, USA), acquiring 8000 events. In order to avoid the experimental error within groups and ensure data consistency in each group, each treatment was repeated twice within an experiment. In order to decrease the experimental error between groups and calculate the S.D. for each group, the experiment was repeated three times.

\section{Construction of luciferase reporter plasmids and dual luciferase activity assay}

The Grb10 3'UTR sequences were amplified by PCR from human genomic DNA using the primers as follow: forward/ Spel: GGCACTAGTCCTCCTTGCCCACTCAGGCT; reverse/ Apal: CATGGGCCCATGGGAAGGACTGGGGTGTC. After being double digested with Spel and Apal, the PCR product was cloned into the downstream of firefly luciferase gene in pGL3 control vector (Promega). Deleting miR199a target sites in the $3^{\prime}$ UTR of Grb10 was used as negative control. All these constructs were verified by DNA sequencing.

For luciferase reporter assays, HEC-1B cells were seeded in 48-well plates and allowed to attach overnight, then transfected with $50 \mathrm{nM}$ of miR199a mimics, miR199a inhibitor, premiR control, or antimiR control (GenePharma Co., Ltd, Shanghai, China) by using lipofectamine 2000 (Invitrogen). HEC-1B cells were also transfected with wild or mutant reporter plasmid. PRL-TK containing Renilla luciferase was co-transfected for data normalization. Two days later, cells were harvested and the luciferase activity was measured with the dual luciferase assay system (Promega). Each treatment was performed in triplicate in three independent experiments. Firefly luciferase activity was normalized to Renilla luciferase activity and the results were expressed as relative luciferase activity (Firefly LUC/Renilla LUC).

\section{Western blot}

Protein lysates from HEC-1B cells were boiled in SDS/ $\beta$-mercaptoethanol sample buffer, and $60 \mu \mathrm{g}$ samples were loaded into each lane of $12 \%$ polyacrylamide gels. The proteins were separated by electrophoresis and transferred to polyvinylidene fluoride membrane (PVDF; Amersham Pharmacia Biotech) by electrophoretic transfer. The membrane was incubated with rabbit anti-GRB10 (Proteintech Group, Inc., Chicago, IL, USA) or -B-ACTIN polyclonal antibody (Santa Cruz Biotechnology, Inc.) overnight at $4{ }^{\circ} \mathrm{C}$. The specific proteinantibody complex was detected by using HRP-conjugated goat anti-rabbit IgG (Jackson Immunoresearch Laboratories, Inc., West Grove, PA, USA). Detection by the chemiluminescence reaction was executed using the ECL kit (Millipore, Billerica, MA, USA). B-ACTIN was used as a loading control. The experiment was repeated at least three times. The bands were analyzed using Quantity One analyzing system (Bio-Rad).

\section{Immunohistochemistry}

Sections $(4 \mu \mathrm{m})$ of uterine tissues were deparaffinized in xylene and rehydrated in descending ethanol series. Antigen retrieval was accomplished through microwave irradiation of the sections in $10 \mathrm{mM}$ sodium citrate buffer. Slides were incubated with rabbit anti-GRB10 polyclonal antibody (Proteintech Group, Inc.), then incubated with HRP-conjugated goat antirabbit IgG (Jackson Immunoresearch Laboratories). The antibody stains were developed by the addition of diaminobenzidine (DAB; Sigma-Aldrich) and the nuclei were stained with hematoxylin (Sigma-Aldrich). The sections were incubated with normal goat serum as negative control. Samples were viewed under Nikon TE 2000-U microscope (Nikon).

\section{Statistical analysis}

There were at least three rats in each treatment group. The results of northern blot and in situ hybridization were repeated three times. All values were reported as the mean \pm s.E.M. Statistical analysis was done using one-way ANOVA. When significant effects of treatments were indicated, the Student-Newman-Keuls multi-range test was applied using SPSS version 13.0 (SPSS, Inc.). $P<0.05$ was considered statistically significant.

\section{Results}

\section{MiR199a expression and regulation in rat uterus during early pregnancy}

In this study, we first examined the expression pattern of miR199a in rat uterus during the peri-implantation period by northern blot. The expression levels of
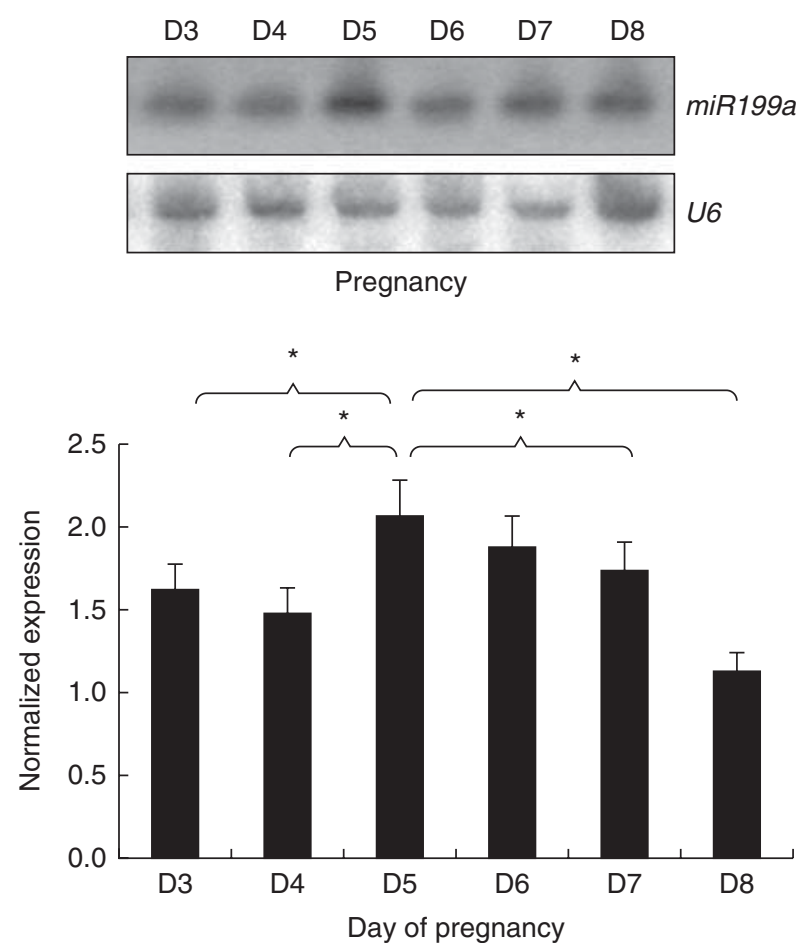

Figure 1 The dynamics of miR199a expression in rat uterus during early pregnancy. The expression of miR199a in rat uteri from day 3 to day 8 of pregnancy was detected by northern blot. Hybridization was conducted with a ${ }^{32}$ P-labeled probe specific for miR199a or U6. U6 snRNA was used as a loading control to normalize for gel loading and transfer. The histogram represents the miR199a levels quantified by densitometric analysis and expressed as a ratio of miR199a to U6 intensity. ${ }^{*} P<0.05$. 
A

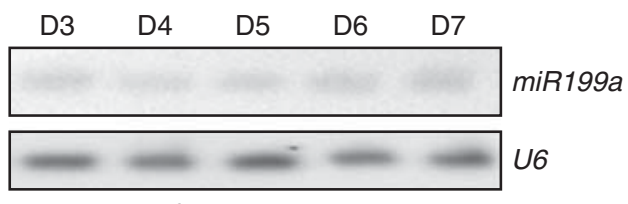

Day of pseudopregnancy

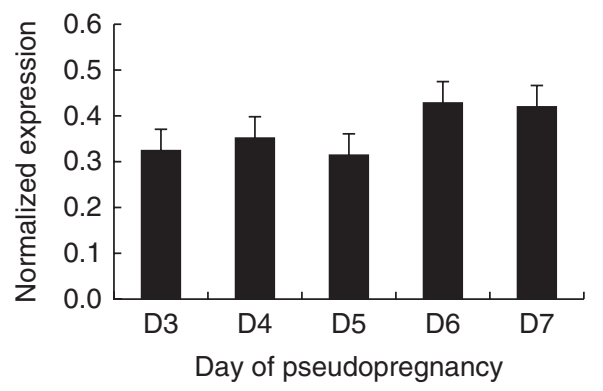

B
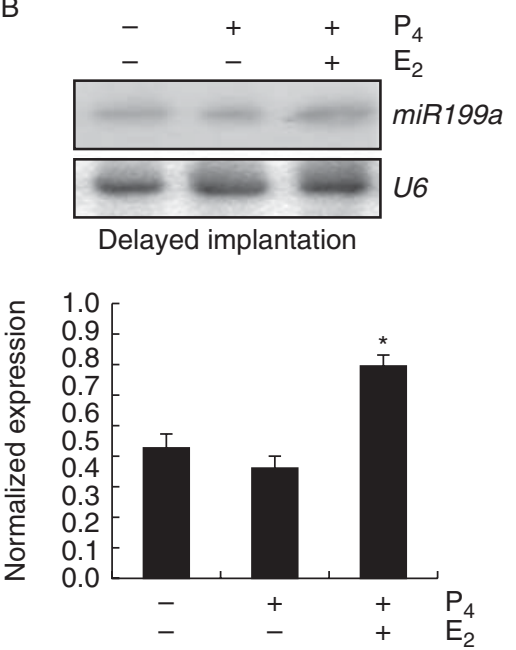

C
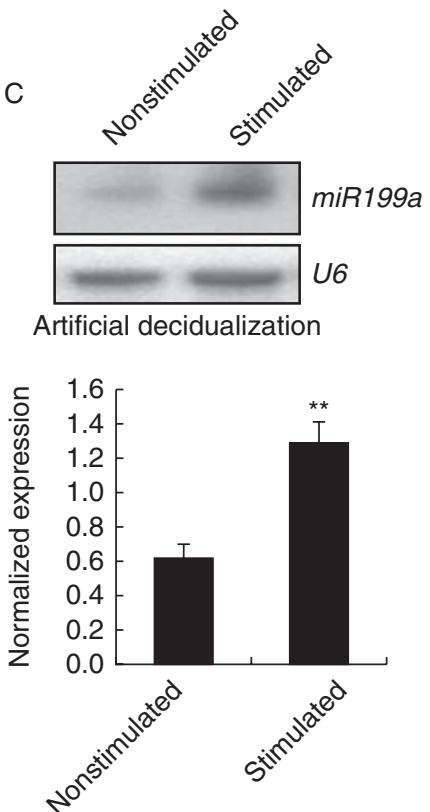

Figure 2 The expression pattern of miR199a in rat uteri in the model of pseudopregnancy, delayed implantation, and artificial decidualization. The expression of miR199a in rat uteri in the model of pseudopregnancy (A), delayed implantation (B), and artificial decidualization (C) was detected by northern blot. Hybridization was conducted with a ${ }^{32}$ P-labeled probe specific for miR199a or U6. U6 snRNA was used as a loading control to normalize for gel loading and transfer. The histogram represents the miR199a levels quantified by densitometric analysis and expressed as a ratio of miR199a to U6 intensity. ${ }^{*} P<0.05,{ }^{* *} P<0.01$.

miR199a on g.d.5-6 were higher than on g.d.3-4 and g.d.7-8 $(P<0.05$; Fig. 1).

To understand whether miR199a expression was dependent upon the presence of embryos, the expression of miR199a in uteri from day 3 to day 7 of pseudopregnancy was detected by northern blot (Fig. 2A). Low levels of miR199a were detected in the uteri on day 3 of pseudopregnancy. The expression levels of miR199a in the uteri from day 4 to day 7 of pseudopregnancy were similar to those on day 3 of pseudopregnancy, implying that miR199a expression was not dependent upon the presence of embryos.

To detect whether miR199a expression was dependent upon embryo status, activation of a delayed implantation model was used for northern blot analysis. The ovariectomized pregnant rats were injected with progesterone to maintain delayed implantation. The delayedimplantation rats were treated with $17 \beta$-estradiol to terminate delayed implantation and activate the blastocysts. Low levels of miR199a were found in the uterus under the delayed implantation condition, but the miR199a levels were significantly enhanced after implantation was activated with estrogen treatment $(P<0.05$; Fig. 2B), suggesting that miR199a expression was dependent upon activated blastocysts, not upon dormant blastocysts.

To test whether miR199a expression was regulated by decidualization, a model of experimentally induced decidualization was used for northern blot analysis. The expression levels of miR199a in the decidualized uterus were higher than those in the nonstimulated uterus on day 7 of pseudopregnancy ( $P<0.01$; Fig. $2 \mathrm{C}$ ).

\section{The effects of steroid hormones on miR199a expression}

The effects of steroid hormones on miR199a expression were examined by northern blot (Fig. 3) analysis. Low levels of miR199a were detected in the ovariectomized rat uterus. Progesterone treatment slightly increased the expression of miR199a. Treatment with $17 \beta$-estradiol significantly boosted miR199a expression $(P<0.05)$. Treatment with $17 \beta$-estradiol and progesterone further enhanced miR199a levels $(P<0.01)$.

\section{Localization of miR199a in rat uteri}

Localization of miR199a in rat uterus during early pregnancy was detected by in situ hybridization. MiR199a was mainly located in the superficial and deep stroma and the circular and longitudinal smooth muscle on g.d.4 (Fig. 4A). On g.d.5 and g.d.6, positive signals of miR199a were strengthened in the superficial and deep stroma or the primary and secondary decidual zone (Fig. 4B and C). In the model of delayed implantation, weak miR199a staining was found in the superficial stroma under delayed implantation conditions (Fig. 4D and E). After delayed implantation was terminated by estrogen treatment and embryos began to implant, strong miR199a signals were detected in the 

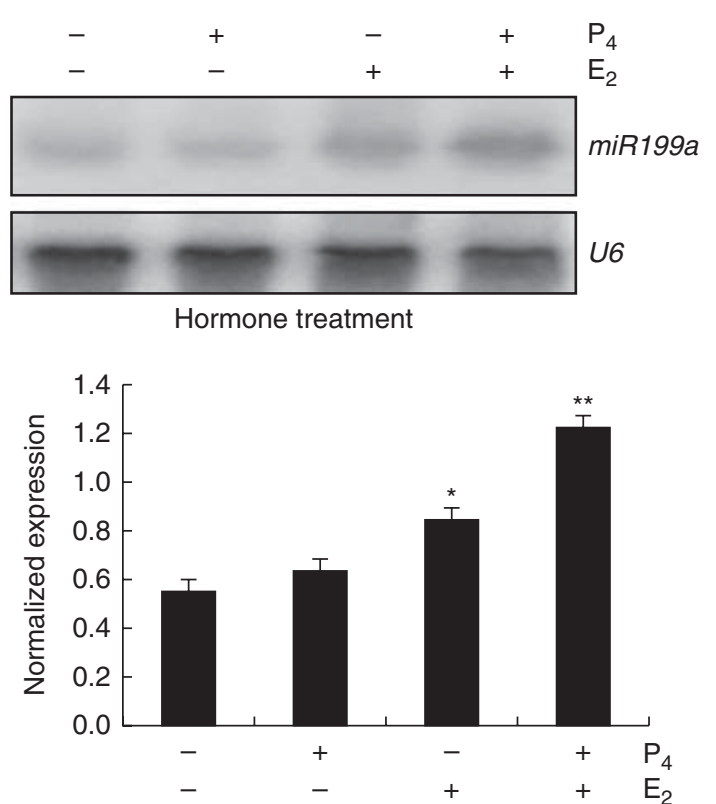

Figure 3 The effects of steroid hormones on miR199a expression in rat uterus. The effects of steroid hormones on miR199a expression in uteri of ovariectomized rats were detected by northern blot. Hybridization was done with a ${ }^{32} \mathrm{P}$-labeled probe for miR199a or U6. U6 snRNA was used as a loading control to normalize for gel loading and transfer. The histogram represents the miR199a levels quantified by densitometric analysis and expressed as a ratio of miR199a to U6 intensity. ${ }^{*} P<0.05,{ }^{* *} P<0.01$.

superficial stroma (Fig. 4F). In the model of artificial decidualization, weak staining was found in the superficial and deep stroma in the control uterine horn on day 7 of pseudopregnancy (Fig. 4G). However, in the oil-infused uterus, strong miR199a signals were found in the primary and secondary decidual zone and myometrium (Fig. 4H).

\section{The effects of miR199a on cell proliferation}

The effects of miR199a on cell proliferation were detected by MTS assay. In order to detect the alteration of cell proliferation, ESCs were transfected with miR199a mimics or inhibitor (Fig. 5A). The relative proliferation rates in ESCs transfected with miR199a mimics were significantly diminished compared with premiR control $(P<0.05)$. The relative proliferation rates in cells transfected with miR199a inhibitor were obviously augmented compared with antimiR control $(P<0.05)$. These results show that overexpression of miR199a inhibits cell proliferation, while low expression of miR199a promotes cell viability.

\section{The effects of miR199a on cell apoptosis}

To further explore the role of miR199a in controlling cell growth, apoptosis in ESCs was determined by flow cytometry analysis (Fig. 5B). Compared with premiR control, transfection with miR199a mimics enhanced the number of early (7.37 vs $1.47 \%$ ) and late apoptotic cells $(11.30$ vs $3.14 \%)$. When cells were transfected with miR199a inhibitor, the number of early apoptotic cells was slightly reduced ( 1.75 vs $2.71 \%$ ), and the number of late apoptotic cells was observably lessened compared with antimiR control ( 2.10 vs $4.64 \%)$.

\section{Prediction and confirmation of the target gene of miR199a}

To analyze the molecular mechanisms in which miR199a was implicated, we explored its target gene. An online search of miR199a targets by miRanda (http://cbio.mskcc.org/cgi-bin/mirnaviewer/mirnaviewer.pl)
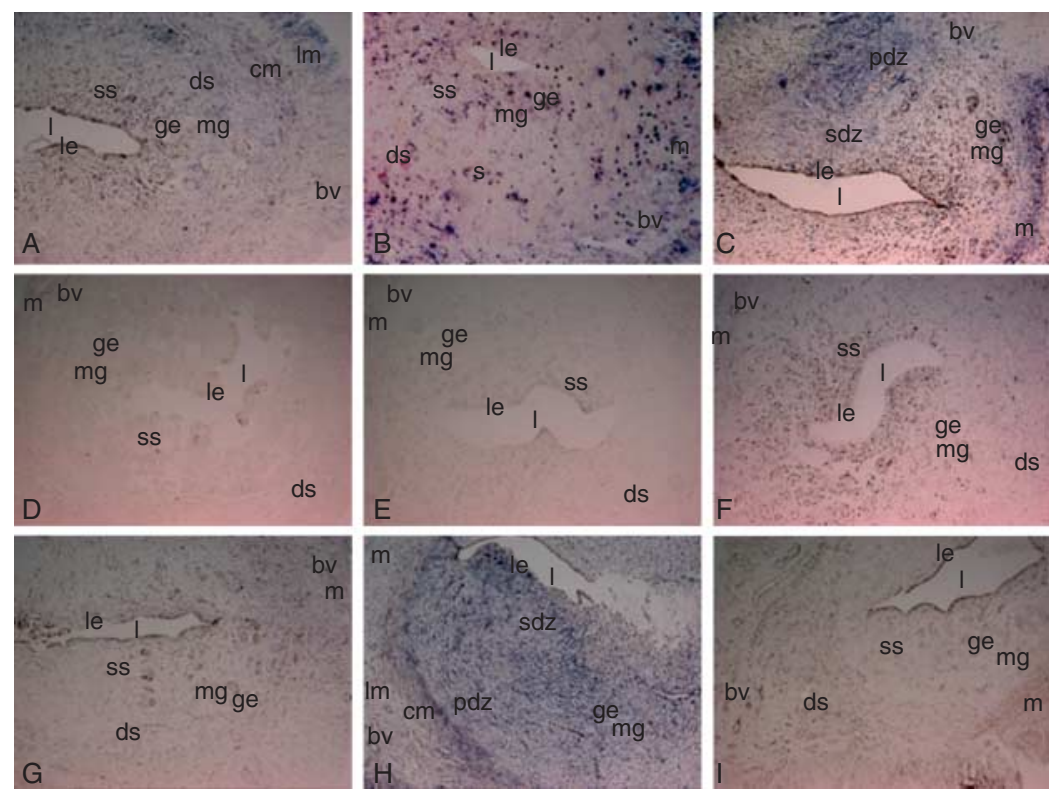

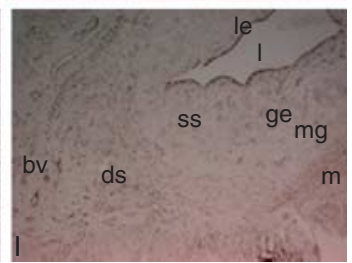

Figure 4 In situ localization of miR199a in rat uterus. Uteri sections from day $4(\mathrm{~A})$, day $5(\mathrm{~B})$, day $6(\mathrm{C})$ of pregnancy, delayed implantation ( $\mathrm{D}$ and $\mathrm{E})$ and activation of delayed implantation (F), and nonstimulated $(\mathrm{G})$ and stimulated $(\mathrm{H})$ artificial decidualization were subjected to in situ hybridization using a DIG-labeled LNA probe specific to miR199a.

To evaluate the specificity of the probe, negative control staining was performed by substituting a DIGlabeled LNA-scrambled probe for the DIG-labeled LNA-miR199a probe (I). Blue staining indicates hybridization signals. The photographs are shown at $\times 100$ original magnification. I, uterine lumen; le, luminal epithelium; $\mathrm{mg}$, maternal glands; ge, glandular epithelium; ss, superficial stroma; ds, deep stroma; bv, blood vessels; m, myometrium; $\mathrm{cm}$, circular smooth muscle; Im, longitudinal smooth muscle; pdz, primary decidual zone; sdz, secondary decidual zone. 

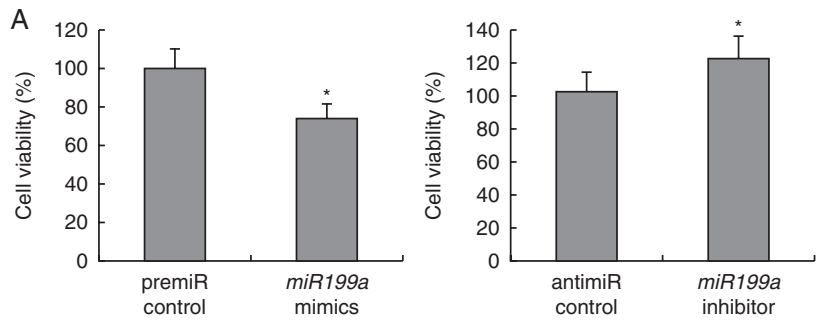

B

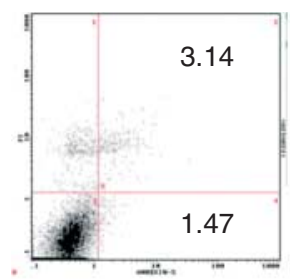

premiR control

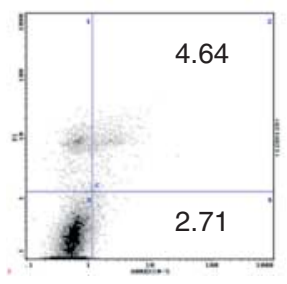

antimiR control

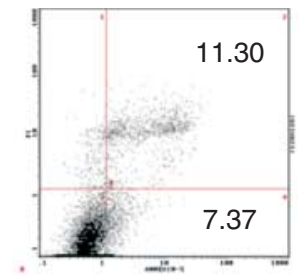

miR199a mimics

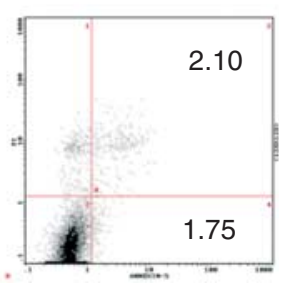

miR199a inhibitor

Figure 5 The effects of miR199a on proliferation and apoptosis of ESCs. ESCs were transfected with premiR control, miR199a mimics, antimiR control, or miR199a inhibitor respectively. After $48 \mathrm{~h}$ of transfection, cell proliferation was determined by MTS assay (A). All experiments were performed at least three times and calculated cell proliferation as stimulation index (SI) (ratio of A570 nm with miR199a mimics vs premiR control or miR199a inhibitor vs antimiR control). Cell apoptosis was determined by flow cytometry (B). The average percentage of apoptosis cells was analyzed in cells transfected by miR199a mimics and inhibitor at early and late stages. Lower left quadrant, viable cells (annexin V-FITC and PI negative); lower right quadrant, early apoptotic cells (annexin V-FITC positive and PI negative); upper right quadrant, late apoptosis/necrosis cells (annexin V-FITC and PI positive). The average percentage of early and late apoptotic cells (representatives of three separate experiments) was shown in the lower right and upper right panels respectively. ${ }^{*} P<0.05$.

TargetScan databases (http://genes.mit.edu/targetscan. test/ucsc.html), and PicTar (http://pictar.mdc-berlin.de/) showed that Grb10 was a putative mRNA target of miR199a. TargetScan, miRanda, and PicTar predictions showed that there was one conservative miR199a-responsive element in the 3'UTR of Grb10 (Fig. 6A).

To validate Grb10 as the target of miR199a, we set up a luciferase reporter assay. The 3'UTR fragment of wt-Grb10 of rat containing the binding sites of miR199a was cloned downstream of the firefly luciferase (designated as wt-Grb10) for the dual luciferase assay (Fig. 6B). Wt-Grb10s were co-transfected with miR199a mimics or inhibitor. Compared with premiR control, the luciferase activity in HEC-1B cells co-transfected with miR199a mimics and wt-Grb10 was decreased by

about $43.19 \%(P<0.01)$. Furthermore, the luciferase activity was upregulated by about $33.43 \%$ in cells co-transfected with miR199a inhibitor and wt-Grb10 compared with antimiR control $(P<0.01)$. These results indicate that miR199a dysregulation affects the binding of miR199a and the 3'UTR of Grb10, leading to the alteration in Grb10 translation.

Base deletion of the seed region was also performed to further confirm the binding site for miR199a in the 3'UTR of Grb10 (Fig. 6C). Deletion of the putative miR199a binding region in the 3'UTR of Grb10 (designated as del-Grb10) was used as control. The luciferase activity was decreased by $80.66 \%$ in the cells co-transfected with miR199a mimics and wt-Grb10 compared with del-Grb10 $(P<0.01)$. These data indicate that miR199a may restrain target gene expression through binding to the seed sequence in the 3'UTR of Grb10.

\section{MiR199a regulates endogenous GRB10 expression}

To ascertain the effects of miR199a on endogenous GRB10 expression, we transfected the HEC-1B cells with miR199a mimics or inhibitor (Fig. 7). Compared with premiR control, GRB10 protein levels were significantly decreased by miR199a mimics $(P<0.05)$. In addition, compared with antimiR control, GRB10 protein levels in the HEC-1B cells were measurably increased by miR199a inhibitor $(P<0.05)$.

\section{The expression of GRB10 in rat uterus}

To further explore the expression profiles of miR199a target gene, Grb10, in vivo, the expression of Grb10 in rat uteri from day 4 to day 6 of pregnancy was detected by immunohistochemistry. On day 4 of pregnancy, strong immunostaining of GRB10 was found in the luminal and glandular epithelium, the superficial stroma, and the myometrium. Moderate intense immunostaining was found in the deep stroma (Fig. 8A). On day 5 of pregnancy, moderate positive hybridization signals were detected in the luminal and glandular epithelium and the myometrium. Weak immunoreaction was found in the stroma (Fig. 8B). On day 6 of pregnancy, moderate immunoreaction was restricted to the luminal and glandular epithelium. Slight immunoreaction was present in the primary and secondary decidual zone and the myometrium (Fig. 8C). These results indicate that the immunostaining of GRB10 in rat uterus on day 4 of pregnancy is stronger than that on day 5 and 6 of pregnancy.

\section{Discussion}

Chakrabarty et al. (2007) reported that miR199a* was differentially expressed in the mouse uterus during 


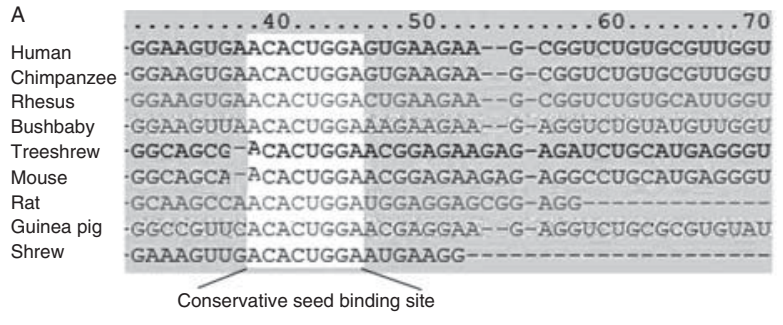

B
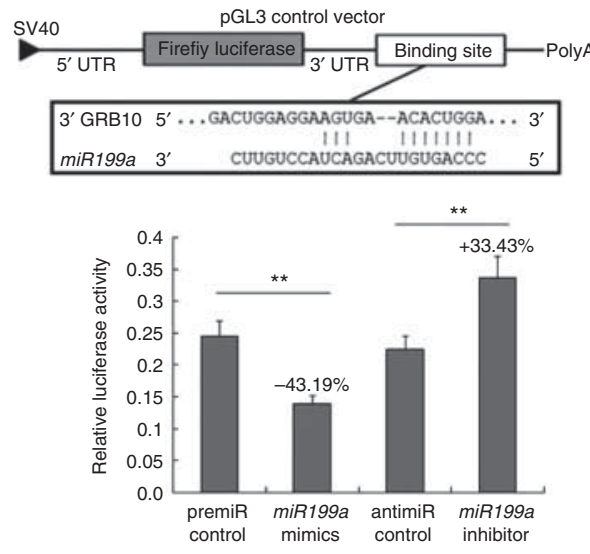

C
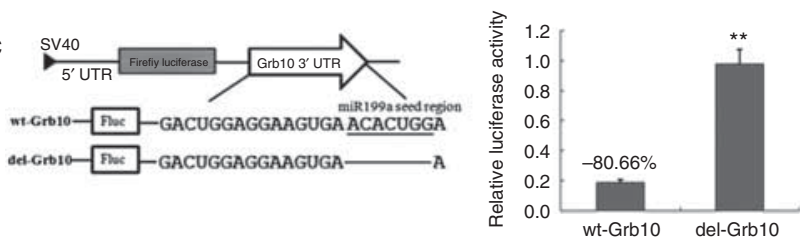

Figure 6 The prediction and confirmation of the miR199a target gene. MiR199a-binding sites in the 3'UTR of Grb10 were compared in crossspecies (A). The $3^{\prime}$ UTR fragment of wt-Grb10 of rat containing the binding sites of miR199a was cloned into the downstream of firefly luciferase reporter gene in $\mathrm{pGL} 3$ control vector (designated as wt-Grb10) for the dual luciferase assay (B). HEC-1B cells were co-transfected with miR199a mimics, premiR control, miR199a inhibitor, or antimiR control, and wt-Grb10 for dual luciferase assay. Deleting the putative miR199a binding region in the 3'UTR of Grb10 (designated as del-Grb10) was used as control. HEC-1B cells were co-transfected with miR199a and wt- or del-Grb10 for the dual luciferase assay (C). pRL-TK containing Renilla luciferase was co-transfected for data normalization. ${ }^{* *} P<0.01$.

implantation. However, the expression pattern and function of miR199a during embryo implantation in rats remained unknown. To study the role of miR199a in embryo implantation, we first examined its spatiotemporal distribution in the rat uterus during the periimplantation period. The expression of uterine miR199a on g.d.5-6 was higher than that on g.d.3-4 and g.d.7-8, and it was mainly located in the stroma or decidua. Embryo transfer experiments have revealed that blastocysts transferred to the uterine lumen of day 4.5 of pseudopregnant recipients remain in a state of dormancy until the endometrium becomes receptive on day 5 , then blastocysts are activated and embryo implantation can be initiated (Psychoyos 1973, Kennedy et al. 2007).
The spatiotemporal distribution of miR199a during early pregnancy suggests that the state of endometrial receptivity and blastocyst activation may promote the expression of miR199a.

To further search whether miR199a expression was related to the induction of implantation, we measured the effects of pseudopregnancy, delayed implantation, and artificial decidualization on miR199a expression. The results showed that miR199a levels were similar in rat uteri from day 3 to day 7 of pseudopregnancy. In the model of activation of delayed implantation, the expression of miR199a was low under delayed implantation conditions, but it was significantly increased after activation of delayed implantation with estrogen treatment. In the model of artificial decidualization, the expression levels of miR199a in the decidualized uterus were significantly enhanced. These results indicate that the increase in miR199a expression relies on activated blastocysts and decidualization, which is coincident with the speculation elicited by the expression pattern of miR199a in early pregnancy.

Progesterone and estrogen are needed for implantation in rats and mice (Rockwell et al. 2002, Chen et al. 2003). Progesterone can help to prepare the uterus for implantation and development of a fertilized egg (Aitken 1978). Estrogen is a critical determinant that specifies the duration of the window of uterine receptivity for implantation (Ma et al. 2003). In this study, we found that miR199a expression was significantly upregulated by $17 \beta$-estradiol or both $17 \beta$-estradiol and progesterone treatment in the ovariectomized rat uterus. The gene expression patterns and hormonal levels were different
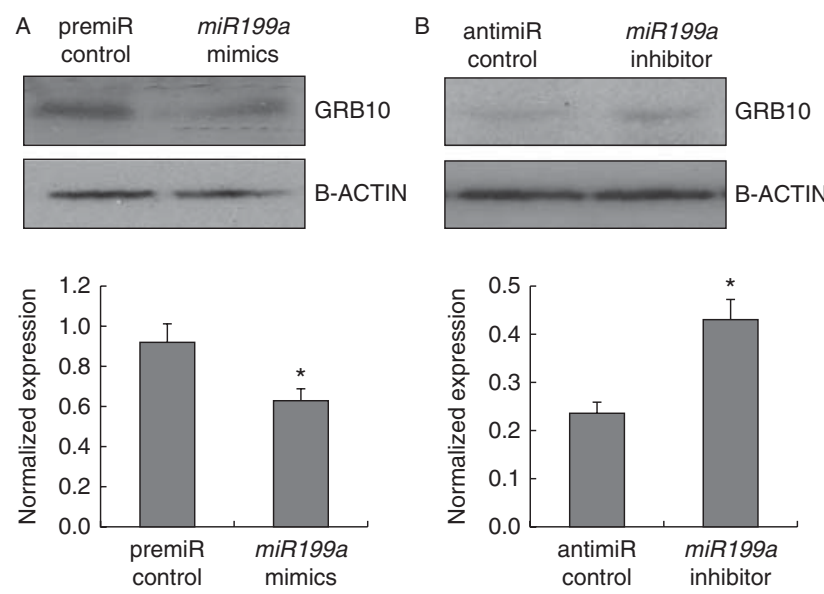

Figure 7 The effects of miR199a on endogenous GRB10 expression. GRB10 protein levels in miR199a mimics (A) and inhibitor (B)-treated HEC-1B cells were detected by western blot. Total protein was separated by SDS-PAGE and then transferred to PVDF membrane. The membrane was incubated with GRB10 or B-ACTIN antibody and detected by the $\mathrm{ECL}$ detection system. The histogram represents the optical densities of the signals quantified by densitometric analysis and represented as GRB10 intensity/B-ACTIN intensity to normalize for gel loading and transfer. ${ }^{*} P<0.05$. 

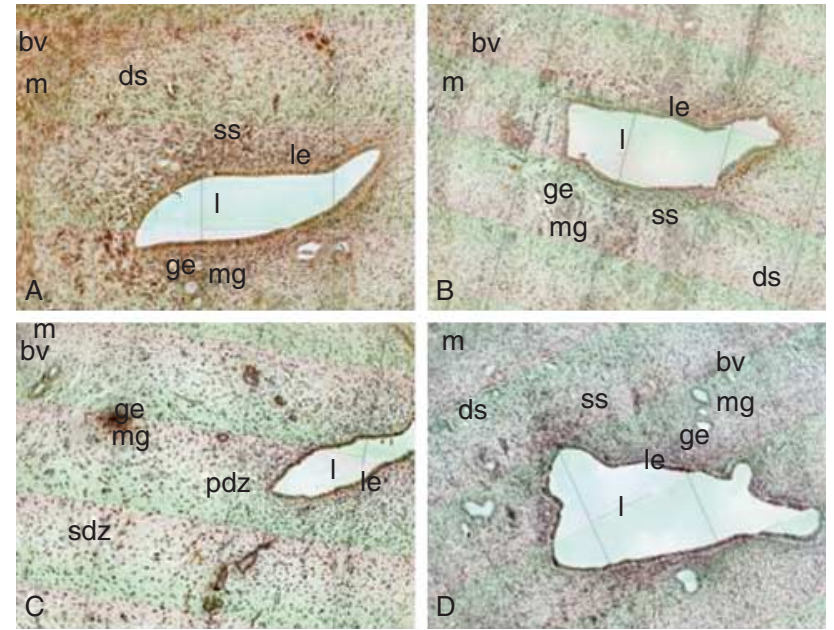

Figure 8 The expression of GRB10 in rat uterus. Uteri sections from day $4(\mathrm{~A})$, day $5(\mathrm{~B})$, and day 6 (C) of pregnancy were subjected to immunohistochemistry using GRB10 antibody. The sections were incubated with normal goat serum as negative control (D). The staining was developed with $D A B$ and the nuclei were stained with hematoxylin. Brown staining indicates positive immunoreaction. The photographs were shown at $\times 100$ original magnification. I, uterine lumen; le, luminal epithelium; mg, maternal glands; ge, glandular epithelium; ss, superficial stroma; ds, deep stroma; bv, blood vessels; m, myometrium; cm, circular smooth muscle; Im, longitudinal smooth muscle; pdz, primary decidual zone; sdz, secondary decidual zone.

between pregnant and nonpregnant rat uterus (Michie \& Head 1994, Shynlova et al. 2007). The expression of miR199a in the uteri shows no comparison between the pregnant rats and the ovariectomized and steroid-treated nonpregnant rats. However, these results indirectly imply that the effects of progesterone and $17 \beta$-estradiol on miR199a expression may be one of reasons that miR199a expression is upregulated during the embryo implantation period.

To explore the possible role of miR199a in rat embryo implantation, we tested the effects of miR199a on the growth of ESCs. Gain of function of miR199a in ESCs isolated from rat uteri inhibited cell proliferation and promoted cell apoptosis. Loss of function of miR199a accelerated cell proliferation and suppressed cell apoptosis. The phenomenon was consistent with the reports of Cheng et al. (2012) and Zhang et al. (2013a) that miR199A suppressed the proliferation of cancerinitiating cells and induced the apoptosis of hepatocellular carcinoma cells in human. Therefore, upregulation of miR199a in rat uteri during the implantation phase may contribute to prevention of the excessive invasion of trophoblastic cells.

To study the possible molecular mechanisms by which miR199a affected cell growth, we analyzed its target gene. An online search of miR199a targets by miRanda, TargetScan, and PicTar showed that there was one conservative miR199a-responsive element in the 3'UTR of Grb10, an imprinted gene. When miR199a mimics or inhibitor were co-transfected with the recombinant vector bearing the putative miR199abinding sites in the 3'UTR of Grb10, luciferase activity was decreased by miR199a mimics and increased by miR199a inhibitor, suggesting that Grb10 should be the target gene of miR199a. A deletion experiment further confirmed that the binding sites in the 3'UTR of Grb10 were specific for miR199a. In addition, overexpression of miR199a reduced the protein levels of GRB10 and knockdown of miR199a promoted the expression of GRB10. These data indicate that Grb10 is not only the target gene of miR199a, but the endogenous GRB10 protein levels are also regulated by miR199a. Disruption of Grb10 in the pancreas enhanced B-cell proliferation and protected mice from streptozotocin-induced B-cell apoptosis (Zhang et al. 2012a). Grb10 could interact with the dynein-binding domain (DBD) of Bim and inhibited apoptosis triggered by overexpression of DBDcontaining Bim isoforms (Hu et al. 2010). Therefore, downregulation of Grb10 by the high levels of miR199a may contribute to promotion of cell proliferation and facilitation of endometrial development.

Although miR199a can regulate GRB10 expression in cell lines, it was unknown whether there was the same condition in the rat uterus. The expression of GRB10 in uteri from day 4 to day 6 of pregnant rats was detected by immunohistochemistry in order to validate the abovementioned results in vivo. Strong immunostaining of GRB10 was found in the stroma on day 4 of pregnancy and weak immunostaining of GRB10 was detected in the stroma or decidua on day 5 and 6 of pregnancy. However, weak staining of miR199a was observed in the stroma on day 4 of pregnancy. On day 5 and 6 of pregnancy, positive signals of miR199a were strengthened in the stroma or decidua. These results show that there is a inverse correlation between the expression of miR199a and GRB10 in the rat uterus.

In conclusion, miR199a expression was increased in rat uterus during the receptive phase and regulated by blastocyst activation and uterine decidualization. Enforced miR199a expression promoted ESC apoptosis, which may be partially through downregulation of Grb10.

\section{Declaration of interest}

The authors declare that there is no conflict of interest that could be perceived as prejudicing the impartiality of the research reported.

\section{Funding}

This work was funded by grants from Natural Science Foundation of China (No. 81370720) and National Research Institute for Family Planning (No. 2012GJSSJKA03), and International S\&T Cooperation Program of China (2012DFB30130). 


\section{References}

Aitken RJ 1978 The hormonal control of implantation. Ciba Foundation Symposia 64 53-83.

Alexander MS, Kawahara G, Motohashi N, Casar JC, Eisenberg I, Myers JA, Gasperini MJ, Estrella EA, Kho AT, Mitsuhashi S et al. 2013 MicroRNA-199a is induced in dystrophic muscle and affects WNT signaling, cell proliferation, and myogenic differentiation. Cell Death and Differentiation 20 1194-1208. (doi:10.1038/cdd.2013.62)

Carson DD, Bagchi I, Dey SK, Enders AC, Fazleabas AT, Lessey BA \& Yoshinaga K 2000 Embryo implantation. Developmental Biology 223 217-237. (doi:10.1006/dbio.2000.9767)

Chakrabarty A, Tranguch S, Daikoku T, Jensen K, Furneaux H \& Dey SK 2007 MicroRNA regulation of cyclooxygenase-2 during embryo implantation. PNAS 104 15144-15149. (doi:10.1073/pnas. 0705917104)

Chen B, Zhang D \& Pollard JW 2003 Progesterone regulation of the mammalian ortholog of methylcitrate dehydratase (immune response gene 1) in the uterine epithelium during implantation through the protein kinase C pathway. Molecular Endocrinology 17 2340-2354. (doi:10.1210/me.2003-0207)

Cheng W, Liu T, Wan X, Gao Y \& Wang H 2012 MicroRNA-199a targets CD44 to suppress the tumorigenicity and multidrug resistance of ovarian cancer-initiating cells. FEBS Journal 279 2047-2059. (doi:10.1111/ j.1742-4658.2012.08589.x)

Granot I, Gnainsky Y \& Dekel N 2012 Endometrial inflammation and effect on implantation improvement and pregnancy outcome. Reproduction 144 661-668. (doi:10.1530/REP-12-0217)

Guo Y, Ying L, Tian Y, Yang P, Zhu Y, Wang Z, Qiu F \& Lin J 2013 miR-144 downregulation increases bladder cancer cell proliferation by targeting EZH2 and regulating Wnt signaling. FEBS Journal 280 4531-4538. (doi:10.1111/febs.12417)

Han ZB, Yang Z, Chi Y, Zhang L, Wang Y, Ji Y, Wang J, Zhao H \& Han ZC 2013 MicroRNA-124 suppresses breast cancer cell growth and motility by targeting CD151. Cellular Physiology and Biochemistry 31 823-832. (doi:10.1159/000350100)

Hu ZQ, Zhang JY, Ji CN, Xie Y, Chen JZ \& Mao YM 2010 Grb10 interacts with Bim L and inhibits apoptosis. Molecular Biology Reports 37 3547-3552. (doi:10.1007/s11033-010-0002-9)

Kennedy TG, Gillio-Meina C \& Phang SH 2007 Prostaglandins and the initiation of blastocyst implantation and decidualization. Reproduction 134 635-643. (doi:10.1530/REP-07-0328)

Koot YE \& Macklon NS 2013 Embryo implantation: biology, evaluation, and enhancement. Current Opinion in Obstetrics \& Gynecology 25 274-279. (doi:10.1097/GCO.0b013e3283630d94)

Ma WG, Song H, Das SK, Paria BC \& Dey SK 2003 Estrogen is a critical determinant that specifies the duration of the window of uterine receptivity for implantation. PNAS 100 2963-2968. (doi:10.1073/pnas. 0530162100)

Michie HJ \& Head JR 1994 Tenascin in pregnant and non-pregnant rat uterus: unique spatio-temporal expression during decidualization. Biology of Reproduction 50 1277-1286. (doi:10.1095/biolreprod50.6.1277)
Pan X, Wang R \& Wang ZX 2013 The potential role of miR-451 in cancer diagnosis, prognosis, and therapy. Molecular Cancer Therapeutics 12 1153-1162. (doi:10.1158/1535-7163.MCT-12-0802)

Psychoyos A 1973 Hormonal control of ovoimplantation. Vitamins and Hormones 31 201-256.

Quinn CE, Simmons DG \& Kennedy TG 2006 Expression of cystatin C in the rat endometrium during the peri-implantation period. Biochemical and Biophysical Research Communications 349 236-244. (doi:10.1016/ j.bbrc.2006.08.054)

Rockwell LC, Pillai S, Olson CE \& Koos RD 2002 Inhibition of vascular endothelial growth factor/vascular permeability factor action blocks estrogen-induced uterine edema and implantation in rodents. Biology of Reproduction 67 1804-1810. (doi:10.1095/biolreprod.102. 006700)

Shynlova O, Tsui P, Dorogin A, Langille BL \& Lye SJ 2007 The expression of transforming growth factor $\beta$ in pregnant rat myometrium is hormone and stretch dependent. Reproduction 134 503-511. (doi:10.1530/ REP-07-0004)

Wang K, Liu F, Zhou LY, Ding SL, Long B, Liu CY, Sun T, Fan YY, Sun L \& Li PF 2013a miR-874 regulates myocardial necrosis by targeting caspase-8. Cell Death \& Disease 4 e709. (doi:10.1038/cddis.2013.233)

Wang X, Zhao Y, Zhang X, Badie H, Zhou Y, Mu Y, Loo LS, Cai L, Thompson RC, Yang B et al. 2013b Loss of sorting nexin 27 contributes to excitatory synaptic dysfunction by modulating glutamate receptor recycling in Down's syndrome. Nature Medicine 19 473-480. (doi:10.1038/nm.3117)

Zhang J, Zhang N, Liu M, Li X, Zhou L, Huang W, Xu Z, Liu J, Musi N, DeFronzo RA et al. 2012a Disruption of growth factor receptor-binding protein 10 in the pancreas enhances $\beta$-cell proliferation and protects mice from streptozotocin-induced $\beta$-cell apoptosis. Diabetes $\mathbf{6 1}$ 3189-3198. (doi:10.2337/db12-0249)

Zhang L, Patterson AL, Zhang L, Teixeira JM \& Pru JK 2012b Endometrial stromal $\beta$-catenin is required for steroid-dependent mesenchymalepithelial cross talk and decidualization. Reproductive Biology and Endocrinology 10 75. (doi:10.1186/1477-7827-10-75)

Zhang J, Wu GQ, Zhang Y, Feng ZY \& Zhu SM 2013a Propofol induces apoptosis of hepatocellular carcinoma cells by upregulation of microRNA-199a expression. Cell Biology International 37 227-232. (doi:10.1002/cbin.10034)

Zhang S, Liu L, Wang R, Tuo H, Guo Y, Yi L, Wang J \& Wang D 2013b MiR199a $5 p$ promotes migration and tube formation of human cytomegalovirus-infected endothelial cells through downregulation of SIRT1 and eNOS. Archives of Virology.

Received 7 July 2013

First decision 29 July 2013

Revised manuscript received 11 October 2013

Accepted 22 October 2013 